\title{
Numerical evaluation of pyramid type sensors for extreme adaptive optics for the European Extremely Large Telescope
}

\author{
Richard M. Clare ${ }^{\mathrm{a}}$, Byron E. Engler ${ }^{\mathrm{a}}$, Steve Weddell $^{\mathrm{a}}$, Iuliia Shatokhina ${ }^{\mathrm{b}}$, Andreas Obereder $^{\mathrm{c}}$, \\ and Miska Le Louarn ${ }^{\mathrm{d}}$ \\ ${ }^{a}$ Department of Electrical and Computer Engineering, Private Bag 4800, Christchurch, New \\ Zealand \\ ${ }^{\mathrm{b}}$ Industrial Mathematics Institute, Altenbergerstraße 69, Linz A-4040, Austria \\ ${ }^{\mathrm{c}}$ MathConsult GmbH, Altenbergerstraße 69, Linz A-4040, Austria \\ ${ }^{\mathrm{d} E u r o p e a n}$ Southern Observatory, Karl-Schwarzschild Straße 2, Garching bei Muenchen, \\ Germany
}

\begin{abstract}
The pyramid wavefront sensor (WFS) is one of a class of WFSs that subdivide the spot at the focal plane into $N$ sectors with an $N$-sided prism, creating $N$ aperture images that can be used to estimate the wavefront aberration. In this paper, we show via end-to-end Monte Carlo simulations that for the European Extremely Largely Telescope (EELT), the pyramid $(N=4)$ and roof WFSs (two orthogonal $N=2$-sided prisms) attain equivalent K band Strehl in the high flux and low read noise regime expected for an extreme adaptive optics (xAO) system. The $N=3$-sided prism is 3-6\% worse in K band Strehl than the pyramid or roof WFSs for this 0 electron read noise case. However, for the low flux ( 5 photons/subaperture/frame) and with a read noise of 5 electrons/pixel/read, the 3 -sided WFS yields a $12 \%$ higher Strehl than a conventional pyramid WFS. In addition, we show that the computationally efficient preprocessed cumulative reconstructor with domain decomposition (pCuReD) yields the same level of $\mathrm{K}$ band Strehl and an indistinguishable PSF profile as a conventional matrix-vector-multiply (MVM) reconstruction for both the pyramid and roof WFSs.
\end{abstract}

Keywords: Adaptive optics, wavefront sensing, pyramid wavefront sensor

\section{INTRODUCTION}

We are currently in the design and early construction period of the Extremely Large Telescope (ELT) era, with the Thirty Meter Telescope (TMT) ${ }^{1}$ Giant Magellan Telescope (GMT) ${ }^{2}$ and European Extremely Large Telescope (EELT) $)^{3}$ all expected to achieve first light in the early years of the next decade. A major science goal of these ELTs is to detect and characterize extra-solar planets.

The resolution of ground-based telescopes is limited by the the earth's time-varying turbulent atmosphere. Adaptive optics (AO) systems will be employed at each of these ELTs ${ }^{1,4,5}$ to overcome the degradatory blurring effect of the atmosphere. Extreme $\mathrm{AO}(\mathrm{xAO})$ systems are very high order AO systems with very high frame rates, and suitable for detection of exo-planets because they can generate high contrast levels between the star and its orbiting planet. Examples of xAO systems are the Gemini Planet Imager ${ }^{6}$ and SPHERE. ${ }^{7}$

The pyramid wavefront sensor (WFS) ${ }^{8}$ is a popular choice for the WFS in AO systems because of its increased sensitivity in closed loop. ${ }^{9,10}$ As shown in Fig. 1, the pyramid WFS consists of a glass prism at the focal plane of the telescope that subdivides the focal plane of the telescope into four quadrants, and a subsequent re-imaging lens produces four aperture images. The pyramid WFS measurements can then be calculated from geometric considerations of these four images. The pyramid WFS is typically modulated by dithering the focal spot around the pyramid apex, which increases its linear range. The pyramid WFS is the current baseline for the Planetary Camera and Spectrograph (PCS), the xAO system for the EELT. ${ }^{11}$

Further author information: (Send correspondence to R.M.C)

R.M.C.: E-mail: richard.clare@canterbury.ac.nz 


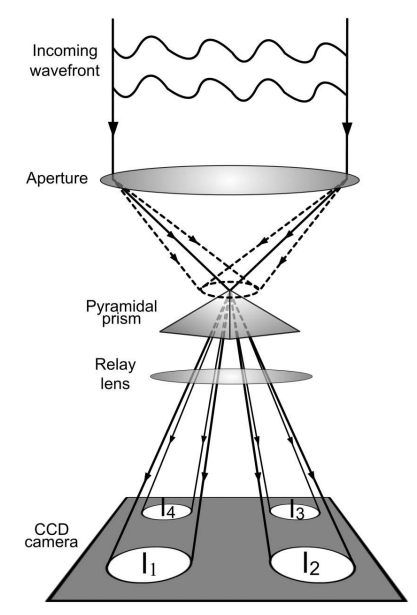

Figure 1. The pyramid ( $N=4$-sided prism) wavefront sensor. ${ }^{12}$ The modulation is shown by the dashed circle over the apex of the pyramid.

In Ref. 13, the authors show that the pyramid WFS is one of a class of WFSs that subdivide the focal plane at the origin into $N$ equal sectors using a prism with $N$ sides. The conventional pyramid WFS is the special case of $N=4$. In order to estimate the wavefront in the orthogonal $x$ and $y$ directions, we must have at least $N=3$ sides. ${ }^{13,14}$ Such 3 -sided prism WFSs, which have subsequently been built by Ref.s 15 and 16 , have the advantage over a traditional 4 -sided prism that they are easier to manufacture, ${ }^{14}$ and they require fewer detector pixels. If we increase the number of sides $N$ in the limit to infinity, we formulate the cone WFS, ${ }^{13}$ also known as the axicon WFS. ${ }^{14,17}$ We can also recover the wavefront phase in the orthogonal $x$ and $y$ directions, by using two 2 -sided prisms or roofs, ${ }^{18}$ with the light on the wavefront sensing path equally split between the two roofs by a beamsplitter. This roof WFS has the advantage that it is easier to manufacture than a pyramid, ${ }^{19}$ and has been shown theoretically to be more sensitive than a pyramid WFS. ${ }^{18,20}$

The conventional approach to reconstructing the phase (or deformable mirror (DM) shape) with a pyramid WFS, or indeed Shack-Hartmann WFS, is to generate an interaction matrix of measurements for a given set of basis modes. The command matrix can then be calculated as a least squares, weighted least squares or maximum a posteriori (MAP) reconstructor from this interaction matrix. The mirror commands are then calculated by multiplying this command matrix by the vector of WFS measurements, hence this reconstruction procedure is known as matrix-vector-multiply (MVM). The MVM reconstruction has a complexity of $O\left(n^{2}\right)$, where $n$ is the number of DM actuators. ${ }^{12}$ For an XAO system on the EELT, there are of the order $10^{3}$ actuators, which need to be controlled at a frequency of typically $3 \mathrm{kHz} .{ }^{12}$ The use of the MVM algorithm on an ELT is not considered computationally feasible with current technology. ${ }^{12}$

A computationally more efficient algorithm for reconstruction of pyramid WFS data is the preprocessed cumulative reconstructor with domain decomposition (pCuReD), which has an $O(n)$ complexity. ${ }^{12}$ The pCuReD algorithm consists of two steps. Firstly, the pyramid WFS measurements are transformed to Shack-Hartmann WFS data. In the second step, the cumulative reconstructor with domain decomposition algorithm ${ }^{21}$ can be applied to this preprocessed data. In Ref. 12, the pCuReD algorithm is shown to provide the same level of correction as the MVM for a pyramid WFS. In this paper, we also apply this algorithm to the roof WFS.

In this paper, we compare the performance of the traditional 4-sided pyramid WFS with the roof WFS and the 3-sided prism WFS using numerical end-to-end Monte Carlo simulations of an XAO system for the EELT. We also compare the traditional MVM reconstruction to the more computationally efficient pCuReD algorithm. The rest of this paper is structured as follows: In Section 2, we outline the mathematical basis for this class of $N$-sided prism WFS, and describe the reconstruction algorithms. In Section 3, we present the simulation results 
for the different WFSs and algorithms. In Section 4, we draw conclusions and discuss future areas of research with these WFSs and algorithms.

\section{METHODOLOGY}

The presence of a prism in the focal plane subdivides the light in the focal plane, which we can describe mathematically as a spatial filter $H(u, v)$. The presence of the relay lens after the prism in the optical path reverses the Fourier transform relationship to produce aperture images in the conjugate aperture plane. The resulting aperture images in the conjugate aperture plane, $I(x, y)$, are given by ${ }^{9}$

$$
I(x, y)=\left|\mathcal{F}^{-1}[H(u, v) \times \mathcal{F}[P(x, y) \exp [j \phi(x, y)]]]\right|^{2},
$$

where $\phi(x, y)$ is the aberrated phase of the wavefront at the aperture plane, and $P(x, y)$ the magnitude of the complex amplitude at the aperture plane. By making use of the convolution theorem and the linearity of the Fourier transform, we can write the aperture images as ${ }^{13}$

$$
I(x, y)=|h(x, y) \odot P(x, y) \exp [j \phi(x, y)]|^{2},
$$

where $\odot$ is the convolution operator, and $h(x, y)$ is the Fourier pair of $H(u, v)$. The aperture images formed from spatial filtering in the focal plane are thus the magnitude squared of the convolution of the complex amplitude at the aperture with the inverse Fourier Transform of the spatial filter. In practice, the prism is modulated by moving the prism (or alternatively the incoming light with a tip-tilt mirror). We denote the instantaneous spatial filter caused by a shift of $\left(u^{\prime}, v^{\prime}\right)$ as $H\left(u-u^{\prime}, v-v^{\prime}\right)$. The inverse Fourier Transform of the shifted spatial filter of a prism is then $h(x, y) \exp \left[j 2 \pi x u^{\prime}\right] \exp \left[j 2 \pi y v^{\prime}\right]$ from the Fourier shift theorem. The aperture images resulting from modulation are the continuous summation of all the aperture images formed over the modulation path $R$

$$
I(x, y)=\oint_{R}\left|h(x, y) \exp \left[j 2 \pi x u^{\prime}\right] \exp \left[j 2 \pi y v^{\prime}\right] \odot P(x, y) \exp [\phi(x, y)]\right|^{2} \mathrm{~d} u^{\prime} \mathrm{d} v^{\prime} .
$$

For the pyramid WFS, as shown in Fig. 1, I(x,y) consists of four aperture images, which we denote as $I_{i}(x, y)$ where $i$ is the image index. The measurements $\left(d_{x}, d_{y}\right)$ for the P-WFS are calculated from the aperture images from geometric considerations,

$$
\begin{aligned}
& d_{x}=\left[I_{1}(x, y)-I_{2}(x, y)-I_{3}(x, y)+I_{4}(x, y)\right] /\left[\sum_{i=1}^{4} I_{i}(x, y)\right] \\
& d_{y}=\left[I_{1}(x, y)+I_{2}(x, y)-I_{3}(x, y)-I_{4}(x, y)\right] /\left[\sum_{i=1}^{4} I_{i}(x, y)\right] .
\end{aligned}
$$

Note that while these measurements were originally considered slope measurements, ${ }^{8}$ they in fact exhibit properties of both slope and direct phase measurements. ${ }^{22}$

The spatial filter for the pyramid WFS, $H_{4}(u, v)$, is defined by

$$
H_{4}(u, v)=\exp [j 2 \pi b(|u|+|v|)]
$$

where $b$ is the slope of the pyramid face. The spatial filters for the two orthogonal $x$ and $y$ roof prisms respectively, $H_{2 x}(u)$ and $H_{2 y}(v)$ are

$$
\begin{aligned}
& H_{2 x}(u)=\exp [j 2 \pi b(|u| \mid)] \\
& H_{2 y}(v)=\exp [j 2 \pi b(|| v \mid)],
\end{aligned}
$$


and the spatial filter for the 3 -sided prism

$$
H_{3}(u, v)=\left\{\begin{array}{cl}
\exp [j 2 \pi b(-2 u)] & -\frac{\pi}{3}<\arctan (u / v)<\frac{\pi}{3} \\
\exp [j 2 \pi b(u-\sqrt{3} v)] & \frac{\pi}{3}<\arctan (u / v)<\pi \\
\exp [j 2 \pi b(u+\sqrt{3} v)] & \text { otherwise. }
\end{array}\right.
$$

These spatial filters $H(u, v)$ for the pyramid, roofs and 3-sided prisms are shown in Fig. 2.

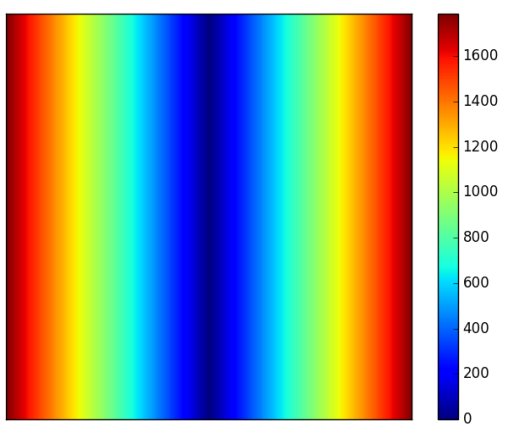

(a)

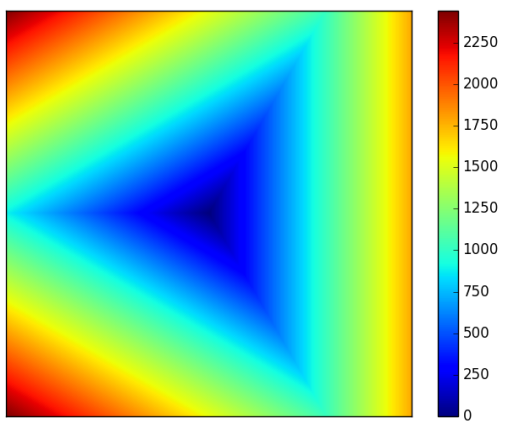

(c)

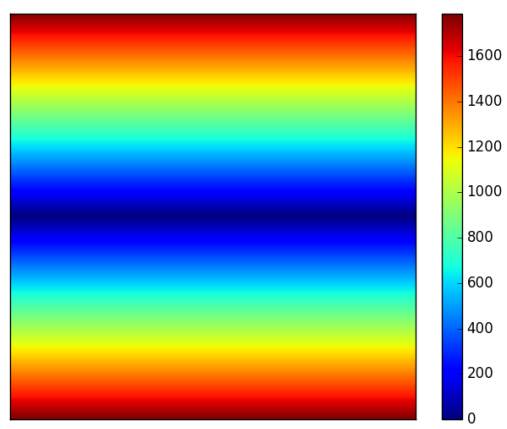

(b)

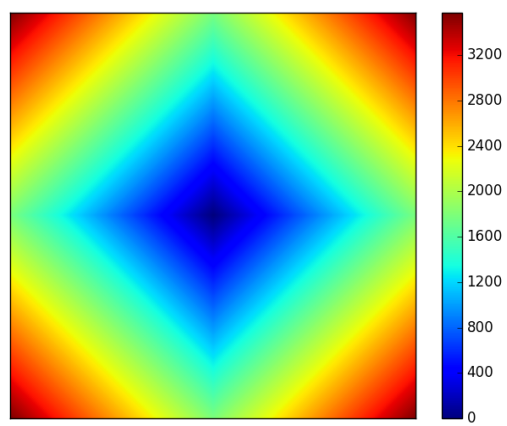

(d)

Figure 2. The prism spatial filter functions $H(u, v)$ at the focal plane (radians) for the (a) $x$-roof $H_{2 x}(u, v)$, (b) $y$-roof $H_{2 y}(u, v)$, (c) 3-sided prism $H_{3}(u, v)$, and (d) pyramid WFS $H_{4}(u, v)$.

The corresponding aperture images for the pyramid, roofs and 3-sided prism WFSs are shown in Fig. 3. These aperture images are calculated for the simulation parameters described in Section 3 using OCTOPUS. ${ }^{24}$ The pyramid WFS consists of four aperture images centred at the vertices of a square. The 3 -sided prism WFS has 3 aperture images located at the vertices of an equilateral triangle. In general, the $N$-sided prism WFS creates $N$ aperture images centred at the vertices of an $N$-sided regular polygon. ${ }^{13}$ The cone WFS produces an annular aperture image. ${ }^{13,14}$

The measurements $\left(d_{x}, d_{y}\right)$ for both the roof WFS and the 3 -sided prism WFS can be calculated from geometric considerations of the aperture images - namely resolving the vectors between the aperture images into their constituent $x$ and $y$ components. For the 3 -sided prism WFS, these measurements are given by

$$
\begin{aligned}
& d_{x}=\left[\frac{3}{2}\left(I_{3}(x, y)-I_{2}(x, y)\right)\right] /\left[\sum_{i=1}^{3} I_{i}(x, y)\right] \\
& d_{y}=\left[\frac{\sqrt{3}}{2}\left(2 I_{1}(x, y)-I_{2}(x, y)-I_{3}(x, y)\right)\right] /\left[\sum_{i=1}^{3} I_{i}(x, y)\right] .
\end{aligned}
$$




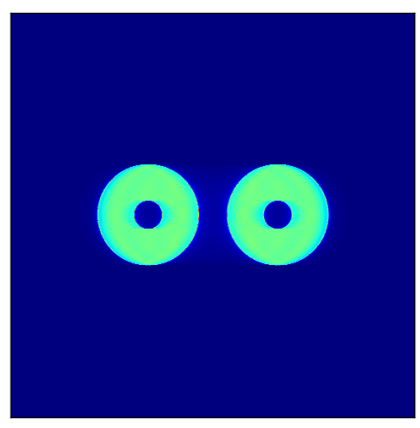

(a)

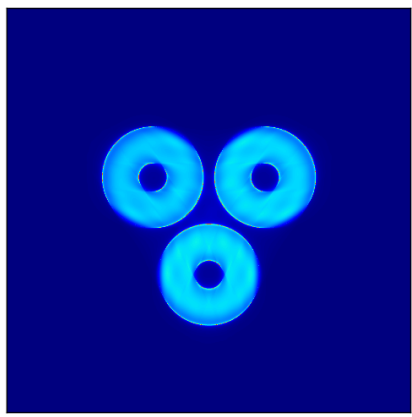

(c)

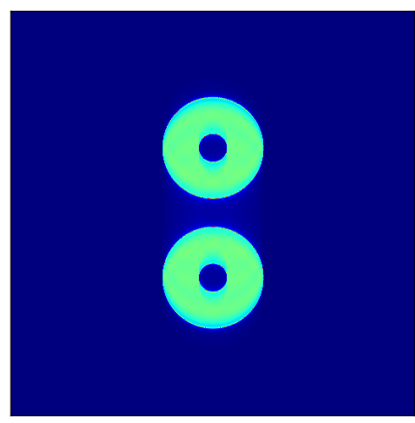

(b)

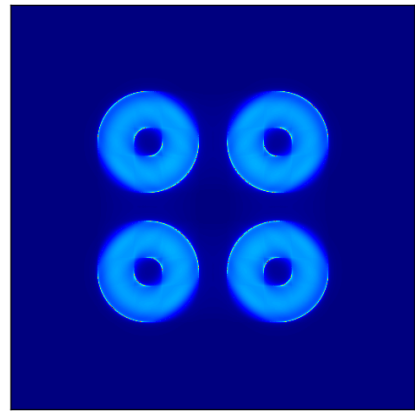

(d)

Figure 3. The aperture images $I(x, y)$ for the (a) $x$-roof, (b) $y$-roof, (c) 3-sided prism, and (d) pyramid WFS.

Similarly, for the roof WFS, the measurements can be expressed by

$$
\begin{aligned}
& d_{x}=\left[I_{x 1}(x, y)-I_{x 2}(x, y)\right] /\left[\sum_{i=1}^{2} I_{x i}(x, y)\right] \\
& d_{y}=\left[I_{y 1}(x, y)-I_{y 2}(x, y)\right] /\left[\sum_{i=1}^{2} I_{y i}(x, y)\right],
\end{aligned}
$$

where the subscripts $x$ and $y$ on the roof aperture images indicate whether the aperture images come from the $x$ roof or $y$ roof. In the following subsection, we discuss reconstructing the wavefront with these pyramid, roof and 3-sided prism WFS measurements.

\subsection{Reconstruction Algorithms}

The wavefront measurements for the pyramid class of WFS are denoted by $d=\left(d_{x}, d_{y}\right)$. The measurements are linearly related to the wavefront $w$ by

$$
d=S w+n,
$$

where $S$ is the interaction matrix, and $n$ is the measurement noise. In practice, the interaction matrix can be measured by applying modes to the DM, and recording the corresponding measurements. For an MVM reconstructor, the estimate of the residual wavefront in the DM space, $\hat{w}$ is given by

$$
\hat{w}=G d,
$$


Table 1. The important atmospheric, telescope, AO and simulation parameters considered in this paper.

\begin{tabular}{cc}
\hline Parameter & Value \\
\hline Fried parameter $\left(r_{0}\right)$ & $12.9 \mathrm{~cm}$ \\
Outer Scale $\left(L_{0}\right)$ & $25 \mathrm{~m}$ \\
Atmosphere & ESO 35 layer model \\
Telescope Diameter $(\mathrm{D})$ & $37 \mathrm{~m}$ \\
Central Obscuration Diameter & $10.4 \mathrm{~m}$ \\
Frame Rate & $3 \mathrm{kHz}$ \\
Delay & $2 \mathrm{Frames}$ \\
WFS Wavelength $\left(\lambda_{W}\right)$ & $700 \mathrm{~nm}$ \\
WFS Order & $148 \times 148 \mathrm{Subapertures}$ \\
Subaperture Width $(d)$ & $25 \mathrm{~cm}$ \\
Prism apex angle & $26.4^{\circ}$ \\
PSF Wavelength & $2.2 \mu \mathrm{m}$ \\
Modulation Width & $4 \lambda_{W} / D$ \\
Time Steps & 1000 \\
\hline
\end{tabular}

where $G$ is the command matrix. In this paper, when we consider the MVM algorithm, we use the MAP reconstructor where $G$ is calculated from the interaction matrix $S$, the covariance of the noise $N=\left\langle n n^{T}\right\rangle$, and the covariance of the turbulent wavefront $W=\left\langle w w^{T}\right\rangle$, by ${ }^{23}$

$$
G=\left(S^{T} N^{-1} S+W^{-1}\right)^{-1} S^{T} N^{-1} .
$$

The controller used for both the MVM and pCuReD in this paper is an integrator, defined by

$$
a_{k}=a_{k-1}+\gamma G d_{k}
$$

where $a_{k}$ is the DM command at time step $k, a_{k-1}$ is the DM command at time step $k-1$, and $\gamma$ is the integrator loop gain.

\section{SIMULATION RESULTS}

In this section, we compare the performance of the pyramid, roof and 3-sided prism WFS using the ESO endto-end AO simulator OCTOPUS. ${ }^{24}$ OCTOPUS is used to generate atmospheric phase screens at each layer of the atmosphere, which are calculated using the $r_{0}$ and $L_{0}$ values in Table 1 . These phase screens are shifted to account for the wind at each layer, and WFS images are calculated with both read and photon noises. OCTOPUS has previously been verified against analytic and other end-to-end simulation codes. ${ }^{23}$

In order to verify that we have correctly set-up our $\mathrm{AO}$ simulation, we calculate the theoretical error due to fitting and temporal errors from the atmosphere and AO system parameters shown in Table 1. The fitting error $\sigma_{\text {fit }}^{2}$ is given by ${ }^{25}$

$$
\sigma_{\text {fit }}^{2}=0.26\left(\frac{d}{r_{0}}\right)^{5 / 3}
$$

and the temporal error $\sigma_{\text {temp }}^{2}$ by ${ }^{25}$

$$
\sigma_{\text {temp }}^{2}=6.88\left(\frac{V \tau}{r_{0}}\right)
$$

where $V$ is the characteristic windspeed and $\tau$ the AO system delay. The total error $\sigma_{\text {tot }}^{2}$, which we can use to check the performance of the numerical simulations in Octopus in the high SNR case, is given by

$$
\sigma_{\text {tot }}^{2}=\sigma_{\text {fit }}^{2}+\sigma_{\text {temp }}^{2}
$$

We note from Fig. 4 that at high SNR the Strehl with the pyramid and roof WFSs match this theoretical Strehl to within $1 \%$. 
The important atmospheric, telescope, $\mathrm{AO}$ and simulation parameters we employ in the simulations are shown in Table 1. The atmospheric parameters $r_{0}$ and $L_{0}$ are chosen for median seeing conditions. The telescope is the $37 \mathrm{~m}$ effective diameter EELT, and we include the central obscuration from the secondary mirror. However, we do not include the spider of metal struts that hold the secondary mirror in place, and causes a reduction in Strehl due to the island effect. ${ }^{26}$ The xAO system we simulate is high order, with $148 \times 148$ subapertures (ie a subaperture width $d$ of $25 \mathrm{~cm}$, and fast, with a frame rate of $3 \mathrm{kHz}$.

In this paper, we only consider a fixed circular modulation of $4 \lambda_{W} / D$, which was found to be optimal in terms of Strehl for a pyramid WFS based EELT XAO system in Ref. 27, in order to constrain the simulation space. Similarly, we only consider a fixed apex angle of the prism $\left(26.4^{\circ}\right)$, which is the same for the pyramid, roofs and 3 -sided prisms, and does not include any overlap of the pupil images. Fauvarque has shown an improvement in sensitivity by choosing an apex angle such that the pupil images overlap, ${ }^{28}$ but we do not investigate this effect in this paper.

In all cases, we modulate the prisms with a circular modulation. The roof prisms could be modulated with a simple 1 dimensional linear modulation instead, but we found that this provided the same Strehl as the circular modulation. For the roof WFS, we do not take into account any transmission losses that would arise at the additional beamsplitter that is required in the optical path compared to a pyramid WFS or 3-sided prism WFS.

We evaluate the performance of the different WFSs and algorithms over a range of guide star fluxes from 5 to $10^{4}$ photons/subaperture/frame. For each WFS, and for each flux level, and for each algorithm it is necessary to optimize the loop gain $\gamma$ in order to have a fair comparison between the WFSs and algorithms. The performance criterion we use to optimize the gain is the on-axis long exposure (LE) Strehl in K band, with a resolution in $\gamma$ of 0.1. We also optimize the regularization in each case, but the Strehl is not as strongly influenced by the regularization as the loop gain.

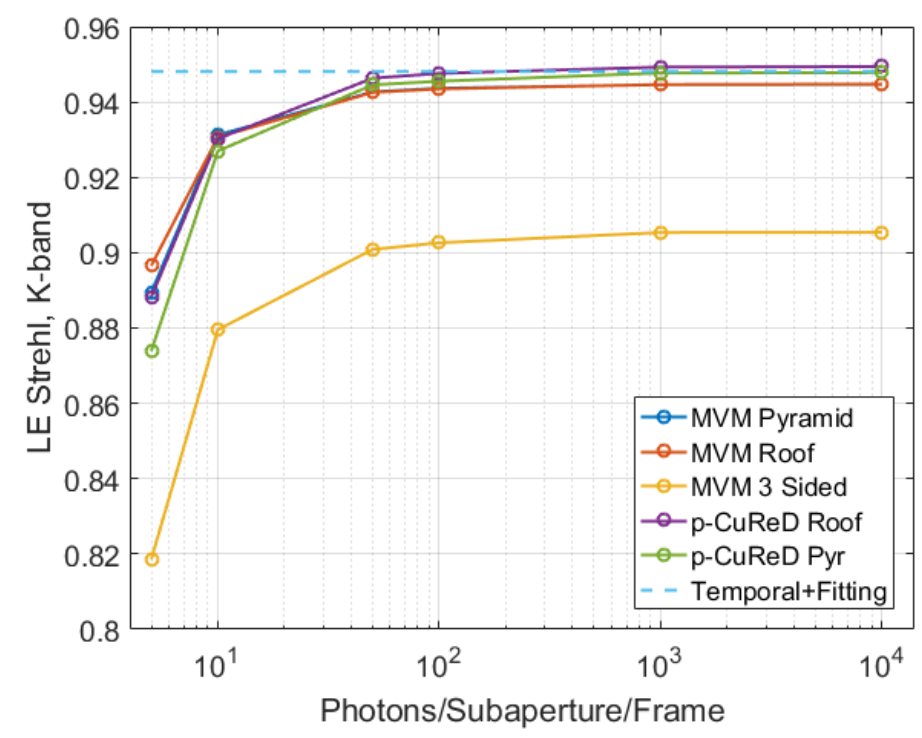

Figure 4. The long exposure Strehl in K band versus the subaperture flux for a read noise of 0 electrons/pixel/read. The dashed line is the $\mathrm{K}$ band Strehl corresponding to the sum of the temporal and fitting errors for this frame rate, delay and atmosphere.

Fig. 4 shows the LE Strehl versus subaperture flux for the three WFSs and two algorithms for the expected case of a read out noise (RON) of 0 electrons/pixel/read. The pCuReD algorithm has not yet been refined for the 3-sided prism WFS case, and so there is no curve for this case. We see that the performance of the pyramid and roof WFS are equivalent over the considered flux range. Similarly, the MVM and pCuReD algorithms yield similar Strehls over the considered flux range for both the pyramid and roof WFSs. The 3-sided prism WFS however, is 3-8\% worse in Strehl than the pyramid or roof WFSs. The 3-sided prism WFS is not as stable in 
closed loop as the pyramid or roof WFSs, and consequently the optimal gains for the 3-sided prism WFS are less than that of the pyramid or roof WFSs. With this lower gain, the temporal error will be greater for the 3 -sided prism than the pyramid or roof WFSs. Additionally, because of the reduced subdivision of the focal plane (into three with the 3-sided prism versus into four with the pyramid or roof WFSs) there may be some unsensed modes with the 3 -sided prism WFS, degrading the Strehl even at high flux.

In Fig. 5, we show the LE Strehl in K band over the same flux levels with the pyramid and 3-sided prism WFS, but with a RON of 5 electrons/pixel/read. The Strehl with the pyramid WFS is higher than that for the 3 -sided prism WFS, except at the lowest SNR point (5 electrons/pixel/read RON, and 5 photons/subaperture/frame) where the 3 -sided prism WFS has a $12 \%$ better correction than the pyramid WFS. Because the 3 -sided prism WFS has fewer detector pixels than the pyramid, by virtue of having three aperture images rather than four, it is less susceptible to read (and photon noise) than the pyramid WFS.

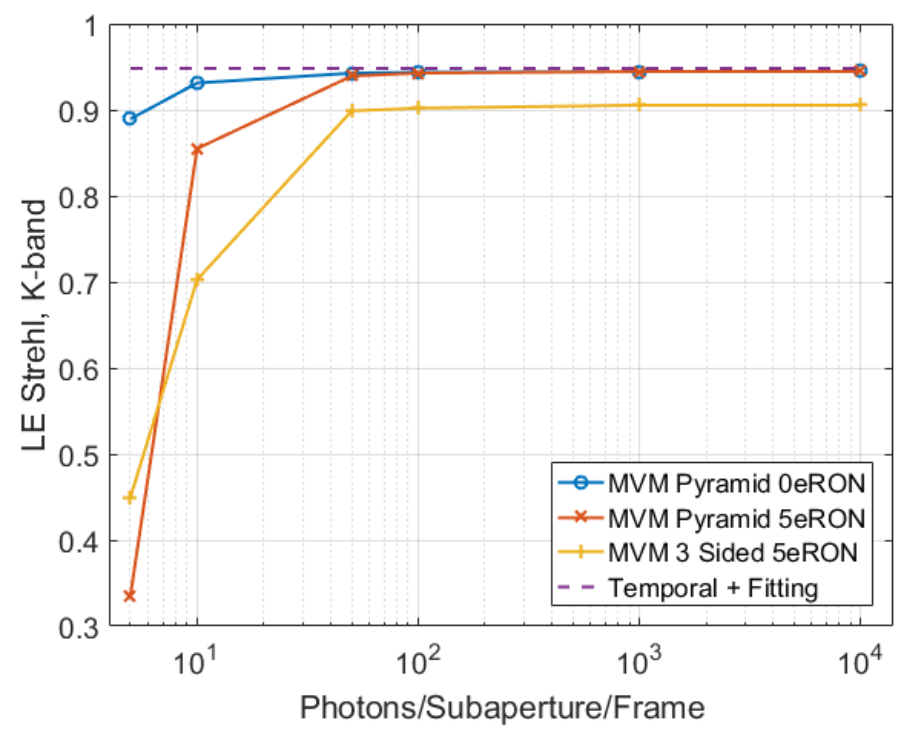

Figure 5. The long exposure Strehl in $\mathrm{K}$ band versus the subaperture flux for a read noise of 5 electrons/pixel/read for the pyramid WFS with the MVM. The dashed line is K band Strehl corresponding to the sum of the temporal and fitting errors for this frame rate, delay and atmosphere. The 0 electrons/pixel/read noise curve us included as a reference.

\subsection{Point Spread Functions}

While in the previous subsection we used K band Strehl as the performance criterion, for an xAO system we are more interested in the ability to detect faint exo-planets, which are off-axis with respect to the parent star. Hence in this subsection we investigate the structure of the point spread functions (PSFs) instead. In Fig. 6, we compare the PSFs for the 3-sided prism, pyramid, and roof WFSs, as well as the MVM and pCuReD algorithms in the highest flux case ( $10^{4}$ photons/subaperture/frame). Additionally, we image the uncorrected (atmospheric) PSF as a reference. In Fig. 6, we see several Airy rings for the 3-sided prism WFS, and significant correction compared to the open loop PSF. There are however, significantly fewer Airy rings present with the 3-sided prism WFS than with either the pyramid or roof WFSs indicating a lower level of correction at higher angles for the 3-sided than the pyramid or roof WFS. The PSFs for the pyramid and roof WFSs in Fig. 6 are visually indistinguishable, for both the MVM and pCuReD algorithms.

In order to further characterize the PSFs for the different WFS and reconstruction algorithms, we plot the radially averaged PSFs - again for the highest flux case - in Fig.s 7 and 8 for the MVM and pCuReD algorithms respectively. Fig. 7 again shows that the 3 -sided prism WFS provides significant correction compared to the open loop atmosphere, but the correction at angles between 200 and 1000 mas is noticeably worse than for the pyramid and roof WFSs. The pyramid and roof WFSs' radial PSF profiles are virtually identical, and we do not suggest there is any significant difference between the two curves. In Fig. 8, we see that the pyramid and 


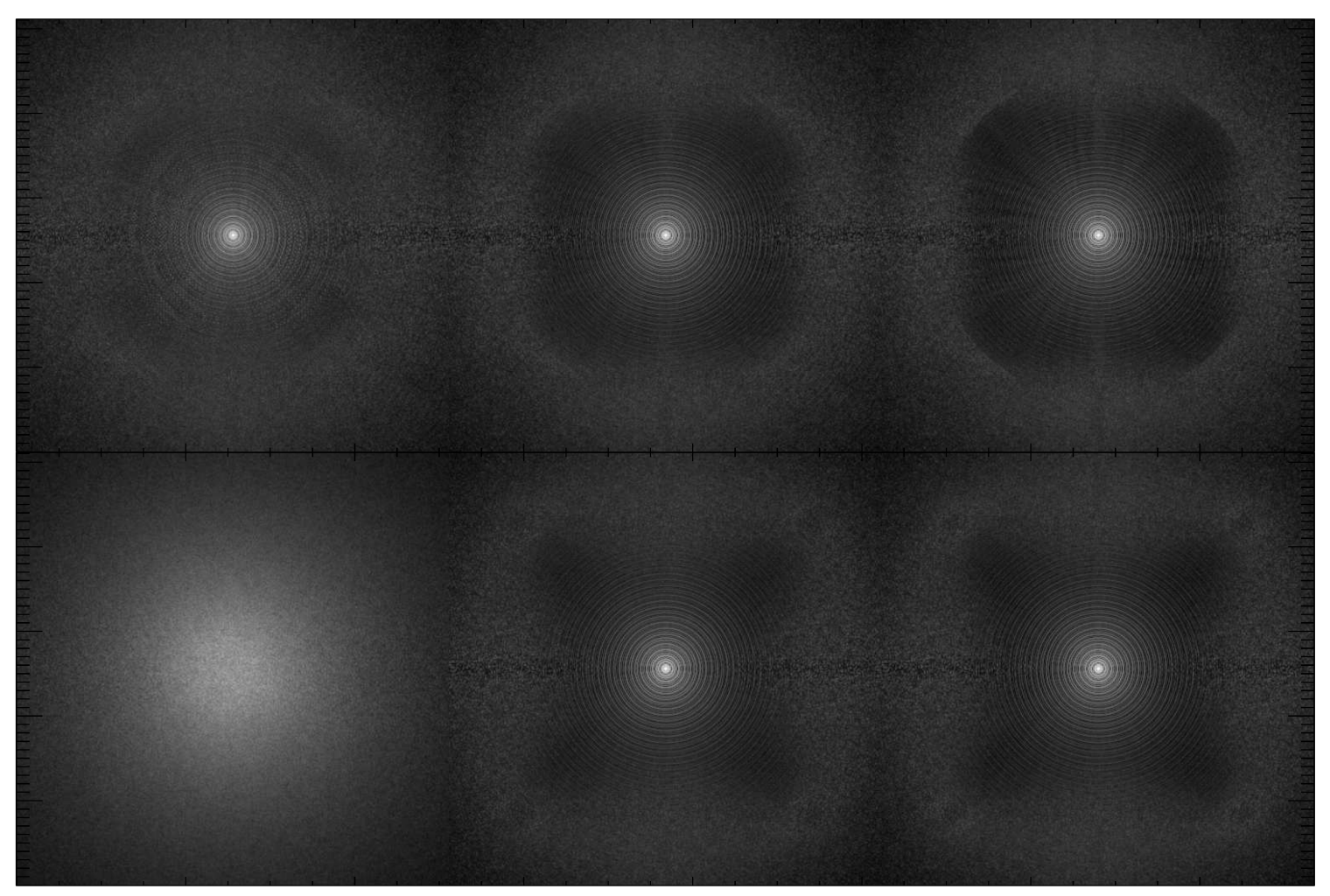

Figure 6. The long exposure PSFs for a flux of $1 \mathrm{e} 4$ photons/subaperture/frame. The top row is a comparison of the three different WFSs with the MVM reconstructor (from left to right: 3-sided, pyramid, and roof WFSs). Bottom left is the uncorrected PSF. Bottom centre and right are the PSFs reconstructed with the pCuReD algorithm for the pyramid and roof WFSs respectively.

roof WFSs' radial PSFs with the pCuReD algorithm are not significantly different from each other, nor from the pyramid WFS using MVM.

\subsection{Convergence}

As well as the performance of the reconstruction algorithm in terms of Strehl or PSF profile, we are also interested in its rate of convergence to the ultimate Strehl. The short exposure Strehl over the first 60 time steps is plotted in Fig. 9 for the high SNR $\left(10^{4}\right.$ photons/subaperture/frame and 0 electrons/pixel RON) case for the pyramid WFS for both the MVM and pCuReD algorithms. We note that while the two algorithms achieve the same ultimate K band Strehl, the MVM algorithm takes approximately 5 iterations more than the pCuReD to reach any given Strehl. This is consistent with a previous comparison between these two algorithms. ${ }^{12}$

\section{CONCLUSIONS}

In this paper, we have shown via numerical simulation that we can achieve high Strehl ratios (at least 90\% Strehl for a median atmosphere in $\mathrm{K}$ band) in an xAO system for the EELT with any of the traditional pyramid WFS, a 3 -sided prism WFS or a roof WFS. The 3-sided prism WFS was 3-6\% worse in Strehl than either the pyramid WFS or roof WFS, which were indistinguishable, across the range of photon flux studied for 0 electrons/pixel of read noise. However, in the noisiest case we studied ( 5 electrons/pixel read noise and 5 photons/subaperture/frame), the 3-sided prism WFS yielded a superior Strehl to the traditional 4-sided pyramid WFS. This improvement at low SNR suggests the 3-sided prism WFS may be better suited to ground layer AO (GLAO) applications than $\mathrm{xAO}$ applications as studied in this paper. The 3 -sided prism WFS also has the advantage that it is easier to manufacture than a pyramid, ${ }^{14}$ requires fewer detector pixels, and does not require an additional beam-splitter as with using two orthogonal roof prisms. 


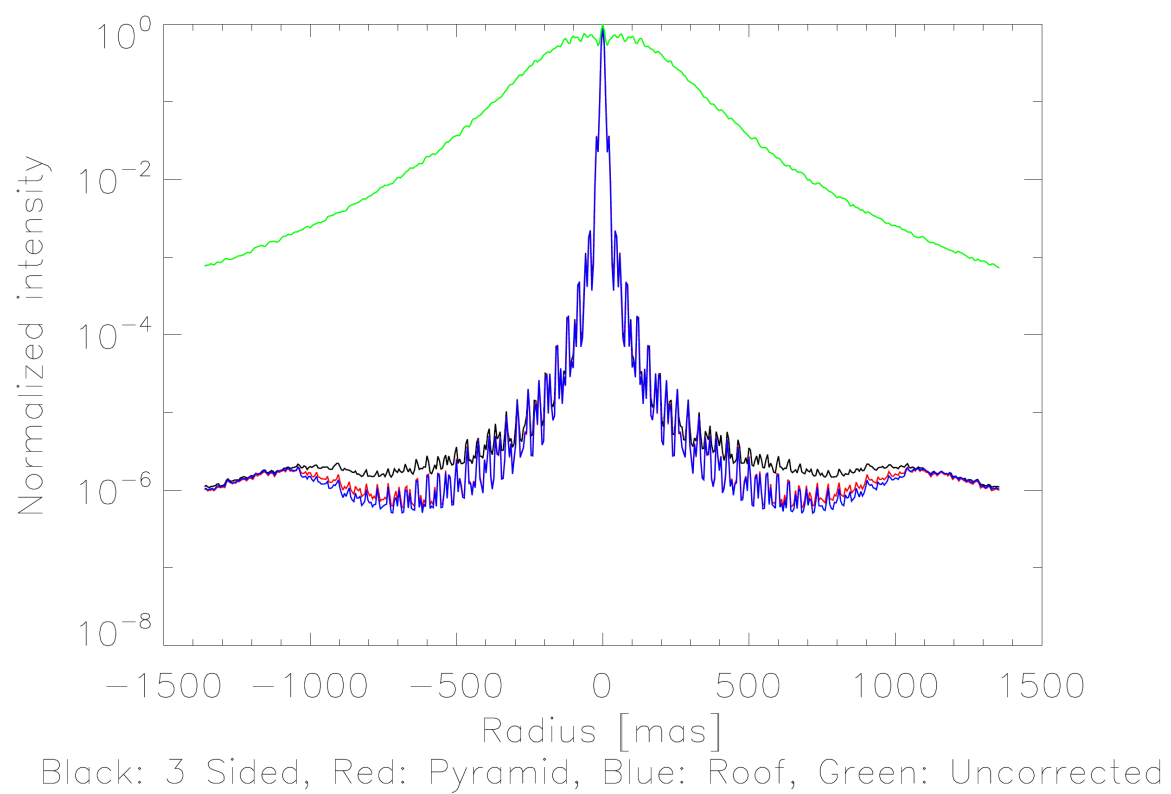

Figure 7. The radially averaged PSFs for a flux of 1e4 photons/subaperture/frame and correction using MVM for the 3-sided (black), pyramid (red), and roof (blue) WFSs. The green curve is the uncorrected PSF.

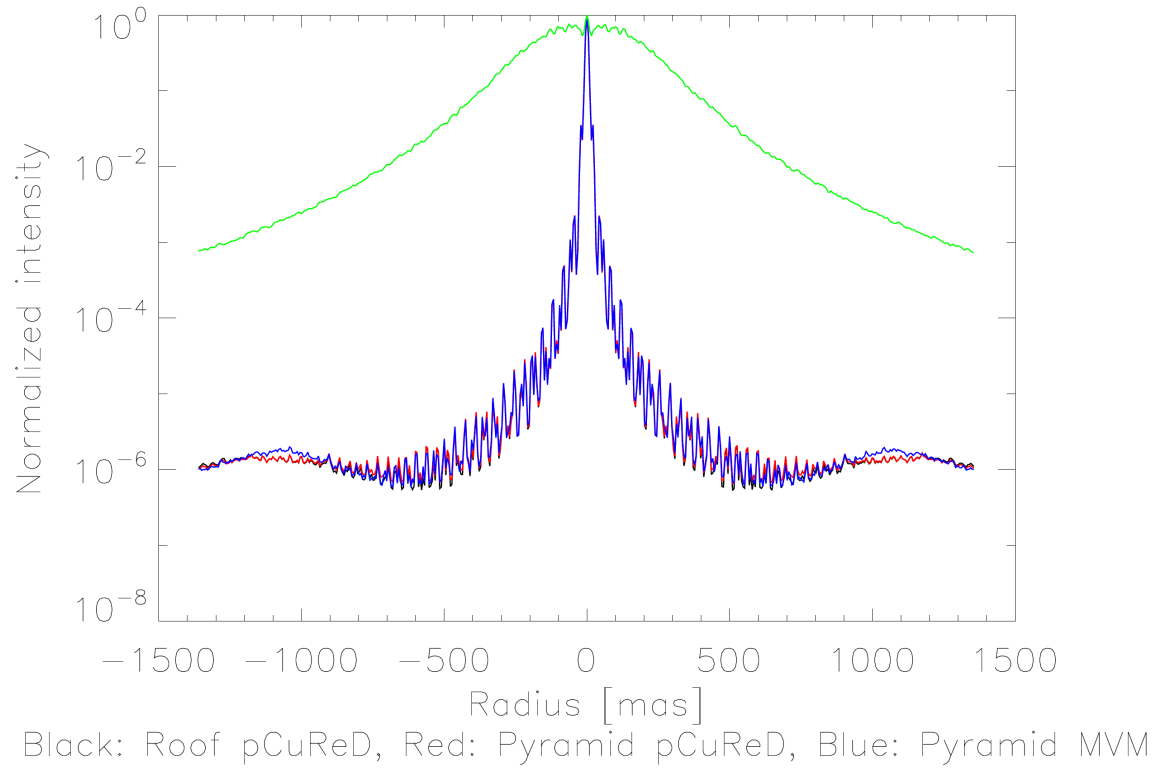

Figure 8. The radially averaged PSFs for a flux of $1 \mathrm{e} 4$ photons/subaperture/frame and correction using pCuReD for the pyramid (red) and roof (black) WFSs. The pyramid WFS with MVM (blue) and the uncorrected PSF (green) are shown for reference.

We have also shown in this paper that the computationally more efficient $\mathrm{pCuReD}$ algorithm can provide a similar level of correction as a traditional MVM reconstructor, both in terms of Strehl and radial PSF profile, for both the pyramid WFS and roof WFS. The pCuReD algorithm also provides a faster convergence to the ultimate Strehl than the MVM.

There is significant scope for future work in comparing $N$-sided prism WFSs for use in the EELT era. In particular, we will also include the cone WFS ( $\infty$-sided) to our comparison of the 3 -sided prism, pyramid and roof. The simulations so far have assumed no telescope spider, which subdivides the pupil images into distinct islands. Inclusion of the spider may change the optimum number of sides of the prism WFS. The pCuReD 


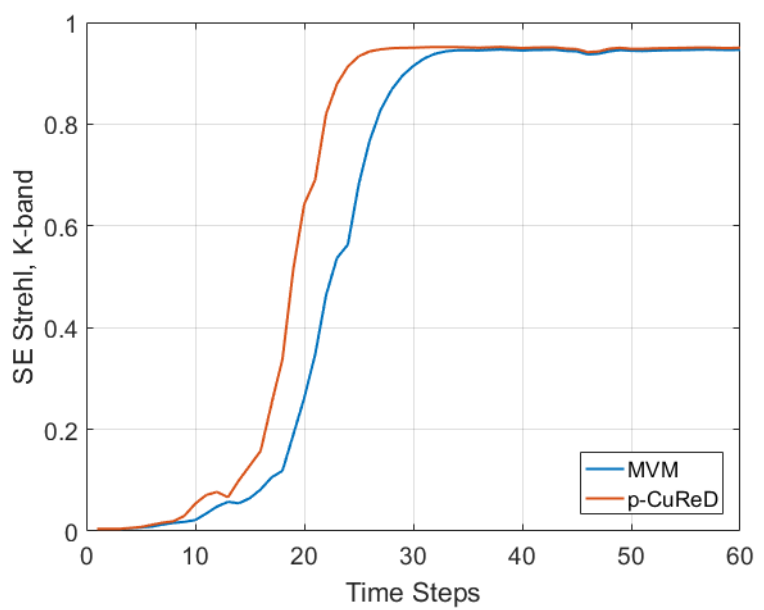

Figure 9. Short Exposure Strehl in K band versus the number of time steps for the pyramid WFS for the MVM (blue) and pCuReD (red) algorithms.

algorithm that we have used in this paper is currently only derived for the pyramid and roof WFSs, not the 3 -sided prsim WFS (nor cone WFS) and this is an area we will investigate in the future. In this paper, we have considered a fixed apex angle for our pyramid-type WFSs, but this key parameter may also play a role on the quality of the reconstruction with this class of WFS, and will be considered in future papers.

\section{REFERENCES}

[1] Boyer, C., "Adaptive optics program at tmt," Third AO4ELT Conference - Adaptive Optics for Extremely Large Telescopes (2017).

[2] Bernstein, R. A., McCarthy, P. J., Raybould, K., Bigelow, B., Bouchez, A. H., Filgueira, J. M., Jacoby, G., Johns, M., Sawyer, D., Shectman, S., and Sheehan, M., "Overview and status of the giant magellan telecsope," Proc. SPIE 9145 (2014).

[3] Tamai, R., Cirasuolo, M., González, J. C., Koehler, B., and Tuti, M., "The e-elt program status," Proc. SPIE 9906 (2016).

[4] Bouchez, A., "Gmt ao system design and status," Third AO4ELT Conference - Adaptive Optics for Extremely Large Telescopes (2017).

[5] Cirasuolo, M., "E-elt design and status," Third AO4ELT Conference - Adaptive Optics for Extremely Large Telescopes (2017).

[6] Macintosh, B., Graham, J. R., Ingraham, P., Konopacky, Q., Marois, C., Perrin, M., Poyneer, L., Bauman, B., Barman, T., Burrows, A. S., Cardwell, A., Chilcote, J., De Rosa, R. J., Dillon, D., Doyon, R., Dunn, J., Erikson, D., Fitzgerald, M. P., Gavel, D., Goodsell, S., Hartung, M., Hibon, P., Kalas, P., Larkin, J., Maire, J., Marchis, F., Marley, M. S., McBride, J., Millar-Blanchaer, M., Morzinski, K., Norton, A., Oppenheimer, B. R., Palmer, D., Patience, J., Pueyo, L., Rantakyro, F., Sadakuni, N., Saddlemyer, L., Savransky, D., Serio, A., Soummer, R., Sivaramakrishnan, A., Song, I., Thomas, S., Wallace, J. K., Wiktorowicz, S., and Wolff, S., "First light of the gemini planet imager," Proceedings of the National Academy of Sciences 111, 12661-12666 (2014).

[7] Kasper, M., Verinaud, C., and Mawet, D., "Roadmap for PCS, the Planetary Camera and Spectrograph for the E-ELT," Proceedings of the Third AO4ELT Conference (2013).

[8] Ragazzoni, R., "Pupil plane wavefront sensing with an oscillating prism," J. Mod. Optics 43, 289-293 (1996).

[9] Esposito, S. and Riccardi, A., "Pyramid wavefront sensor behavior in partial correction adaptive optics," Astron Astrophys 369, L9-L12 (2001).

[10] Chew, T. Y., Clare, R. M., and Lane, R. G., "A comparison of the shackhartmann and pyramid wavefront sensors," Optics Communications 268, 189 - 195 (2006). 
[11] Fusco, T., Sauvage, J.-F., Mouillet, D., Costille, A., Petit, C., Beuzit, J.-L., Dohlen, K., Milli, J., Girard, J., Kasper, M., Vigan, A., Suarez, M., Soenke, C., Downing, M., N'Diaye, M., Baudoz, P., Sevin, A., Baruffolo, A., Schmid, H.-M., Salasnich, B., Hugot, E., and Hubin, N., "SAXO, the SPHERE extreme AO system: on-sky final performance and future improvements," in [Adaptive Optics Systems V], 9909 (2016).

[12] Shatokhina, I., Obereder, A., Rosensteiner, M., and Ramlau, R., "Preprocessed cumulative reconstructor with domain decomposition: a fast wavefront reconstruction method for pyramid wavefront sensor," Appl. Opt. 52, 2640-2652 (2013).

[13] Clare, R. M. and Lane, R. G., "Wavefront sensing from spatial filtering at the focal plane," Proc. SPIE 5169, 43-54 (2003).

[14] Fauvarque, O., Neichel, B., Fusco, T., Sauvage, J.-F., and Girault, O., "General formalism for fourier-based wave front sensing: application to the pyramid wave front sensors," J. Astron. Telesc., Instrum. Syt. 3, 019001 (2017).

[15] Chen, L., Wang, J., Yao, K., Liu, X., Lin, X., Wang, L., and Wang, M., "Experimental demonstration of sequential operation approach for a three-sided pyramid wavefront sensor," IEEE Photonics Journal 8, 1-13 (2016).

[16] Costa, J., Development of a new infrared pyramid wavefront sensor, PhD thesis, University of Heidelberg (2005).

[17] Vohnsen, B., Castillo, S., and Rativa, D., "Wavefront sensing with an axicon," Opt. Lett. 36, 846-848 (2011).

[18] Phillion, D. and K., B., "Two-sided pyramid wavefront sensor in the direct phase mode," Proc. SPIE 6272 (2006).

[19] van Dam, M. A., Conan, R., Bouchez, A. H., and Espeland, B., "Design of a truth sensor for the gmt laser tomography adaptive optics system," Proc. SPIE 8447 (2012).

[20] Wang, J., Bai, F., Ning, Y., Huang, L., and Wang, S., "Comparison between non-modulation four-sided and two-sided pyramid wavefront sensor," Opt. Express 18, 27534-27549 (2010).

[21] Rosensteiner, M., "Wavefront reconstruction for extremely large telescopes via cure with domain decomposition," J. Opt. Soc. Am. A 29, 2328-2336 (2012).

[22] Vérinaud, C., "On the nature of the measurements provided by a pyramid wave-front sensor," Optics Communications 233, 27-38 (2004).

[23] Clare, R. M., Louarn, M. L., and Béchet, C., "Laser guide star wavefront sensing for ground-layer adaptive optics on extremely large telescopes," Appl. Opt. 50, 473-483 (2011).

[24] Le Louarn, M., Verinaud, C., Korkiakoski, V., and Fedrigo, E., "Parallel simulation tools for ao on elts," Proc. SPIE 5490, 705-712 (2004).

[25] Béchet, C., Le Louarn, M., Tallon, M., and Thiébaut, É., "Performances of the fractal iterative method with an internal model control law on the eso end-to-end elt adaptive optics simulator," Proc. SPIE 7015, 70151H-70151H-9 (2008).

[26] Obereder, A., "On the performance of reconstruction methods in the presence of spiders," Third AO4ELT Conference - Adaptive Optics for Extremely Large Telescopes (2017).

[27] Clare, R. M. and Le Louarn, M., "Numerical simulations of an extreme ao system for an elt," Second AO4ELT Conference - Adaptive Optics for Extremely Large Telescopes (2011).

[28] Fauvarque, O., Neichel, B., Fusco, T., and Sauvage, J.-F., "Variation around a pyramid theme: optical recombination and optimal use of photons," Opt. Lett. 40, 3528-3520 (2015). 